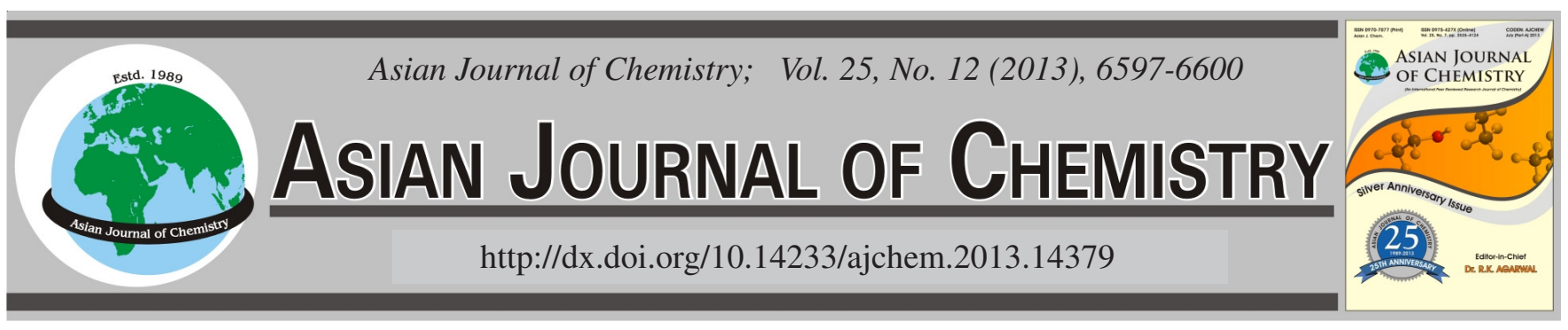

\title{
Antifungal Activity and Mechanism of Major Compound Isolated from Hexane Extract of Curcuma zedoaria
}

\section{Zizhu Chen ${ }^{1, \uparrow}$, Yan Wei $^{2, \dagger}$, Xue Li ${ }^{1}$, Chenchen Peng ${ }^{1}$ and Zhangfu Long ${ }^{1, *}$}

${ }^{1}$ Key Laboratory of Bio-resources and Eco-environment (Ministry of Education), College of Life Sciences, Sichuan University, Chengdu 610064, P.R. China

${ }^{2}$ Department of Viral Encephalitis, National Institute for Viral Disease Control and Prevention, Chinese Center for Disease Control and Prevention, Beijing, 102206, P.R. China

*Corresponding author: Tel/Fax: +86 28 84761793; E-mail: 1zf0028@163.com; zhuzic3672@163.com

$\dagger$ These two authors contributed equally to this work.

\begin{abstract}
The main compound of hexane extract from Curcuma zedoaria was purified using bioactive-guided silica gel column chromatography and high performance liquid chromatography in this study and the structure of the main compound was identified as curcumenol by spectroscopic methods, including high resolution mass spectra (HRMS), FTIR, ${ }^{1} \mathrm{H}$ and ${ }^{13} \mathrm{C}$ NMR spectroscopy. It was firstly authenticated that curcumenol derived from $C$. zedoaria showed higher and broad-spectrum antifungal activity against fungal phytopathogens, such as Fusarium graminearum, Phoma wasabiae, Plasmodiophora brassicae and Magnaporthe grisea while their minimal inhibition concentration were $0.51 .0,1.0,1.0 \mathrm{mg} \mathrm{mL}^{-1}$, respectively. The antifungal activity was not affected by high or low $\mathrm{pH}$ values (from 2-10) or under different temperatures (from $30-80^{\circ} \mathrm{C}$ ). Its antifungal mechanism was further investigated by comparing the ultrastructures of the experimental fungi using scanning electron microscope and transmission electron microscope and results showed that its antifungal activity was realized by means of destroying the cell-wall and influencing the nutrients circulation of the fungal phytopathogens.
\end{abstract}

Key Words: Curcuma zedoaria, Antifungal activity, Curcumenol, Stability, Mechanism.

\section{INTRODUCTION}

Fungal diseases of plants are generally controlled by synthetic chemical fungicides. However, this approach has resulted in the environment polluted heavily while the nontarget organisms and human health concern highlighted the need for the development of new strategies for plant-pathogenic-fungi control ${ }^{1-3}$. It is widely recognized that plants biosynthesize a vast array of secondary metabolites, such as phytoalexins and antifungal compounds for protecting themselves ${ }^{4}$. With this knowledge, lots of researches have been organized to find out the antifungal compounds produced by plants over the past few years ${ }^{5-7}$ and some significant results have been made, such as azadirachtin, carvone and pyrethroids ${ }^{8}$.

Curcuma zedoaria is a traditional Chinese medicine being used to promote blood circulation, relieve pain and eliminate depression. Recently, many reports showed its other functions, such as antioxidative ${ }^{9}$, antiinflammatory ${ }^{10}$, anticancer $^{11}$ and other various beneficial pharmacological activities, it has recently been introduced as dietary supplements and health foods ${ }^{12}$.
The essential oil and extract of $C$. zedoaria has been reported to possess antimicrobial properties ${ }^{13,14}$. In our previous study, $95 \%$ ethanol extract of $C$. zedoaria showed potential activity in vitro models against Phoma wasabiae and three fractions were, respectively extracted by three solvents (different polarities) including hexane, ethyl acetate and methanol while the hexane extract showed high fungicide efficiency against the mycelium growth rates of many phytopathogens, such as Phoma wasabiae, Fusarium graminearum, Sclerotinia sclerotiorum, Penicillium pallidum, Mycogone peniciosa, Botrytis cirerea, Verticillium dahliae ${ }^{15,16}$.

\section{EXPERIMENTAL}

C. zedoaria was collected from Chongzhou, Sichuan, P.R. China, in March, 2010. The specimen was identified by Prof. Xingjin He of Sichuan University, Chengdu, P.R. China and deposited in the Key Laboratory of Bio-resources and Ecoenvironment, Ministry of Education, Chengdu, P.R. China with NO.20100305.

Bioactive-guided isolation: The air-dried and powdered C. zedoaria was macerated with $95 \%$ ethanol and sonicated in a bath sonicator ( $3 \mathrm{~L} \times 1.5 \mathrm{~L}, 20 \mathrm{~min}$ each) under $40 \mathrm{~Hz}$ at 
room temperature. The extract was then filtered and concentrated to extractum using a rotary evaporator and then the extractum was successively partitioned by solid-liquid partition with solvents including hexane, ethyl acetate and methanol, respectively. The excessive solvents in each partitioned fraction were removed by a rotary evaporator.

The hexane extract was further separated using a silica gel column (200-300 mesh) eluted with the solvent mixtures included with petroleum ether and ethyl acetate in four concentration ratios $(\mathrm{w}: \mathrm{w}=16: 1,8: 1,4: 1$ and 1:1) and with methanol at last. Based on thin layer chromatography (TLC) using $\mathrm{GF}_{254}$ and visualization with ultraviolet light $(254 \mathrm{~nm})$, five fractions were obtained, respectively. After that, these five fractions' antifungal activities against $P$. wasabiae were compared by growth rate method described by Liu et al. ${ }^{15}$. Because of the fraction 3 (best fungicide activity) being a mixture, a preparative gradient HPLC was employed in this study to purify pure compounds from it in a Gilson Prep-HPLC system coupled with a Waters $\mathrm{X}$ Bridge Prep- $\mathrm{C}_{18}$ reversed-phase column $(100 \mathrm{~mm} \times 30 \mathrm{~mm}, 5 \mu \mathrm{m})$ maintained at $25^{\circ} \mathrm{C}$. Methanol gradient solutions (from $55-100 \%$, dissolved in doubledistilled $\mathrm{H}_{2} \mathrm{O}$ ) were used as the mobile phase for 8 min and holding at the $100 \%$ concentration for $3.8 \mathrm{~min}$ while the purification process was monitored under UV lights at 214 and $254 \mathrm{~nm}$. The flow velocity was set as $20 \mathrm{~mL} / \mathrm{min}$. When F3 was separated, the compound corresponding to the major peak was collected and the solvents of the mobile phase were removed by evaporation using a rotary evaporator at $35^{\circ} \mathrm{C}$.

The high resolution mass spectra (HRMS) (ESI) were recorded on a Bio TOF IIIQ from Bruker Daltonics Inc. The FTIR spectra were carried out by a Nicolet 6700 Fourier transform spectrometer. The ${ }^{1} \mathrm{H}$ and ${ }^{13} \mathrm{C}$ NMR spectra were obtained in deuterated methanol with a Bruker AV II- $600 \mathrm{MHz}$ NMR spectrometer with tetramethylsilane as an internal standard; chemical shifts are given in $\delta$ (ppm).

Assay for antifungal activity: The major compound's antifungal activities against typical pathogens, including $P$. wasabiae, Fusarium graminearum, Plasmodiophora brassicae and Magnaporthe grisea, were measured by the growth rate method. Briefly, after it was dissolved in acetone, the compound was then mixed with $20 \mathrm{~mL}$ molten potato dextrose agar (PDA) medium to the final concentrations of $0.125,0.25$, $0.5,1.0$ and $2.0 \mathrm{mg} \mathrm{mL}^{-1}$ in $90 \mathrm{~mm}$ diameter sterilised Petri plate $^{6}$ while the plate contained with same volume of acetone and PDA medium used as the control. All experimental and control plates were breeding with a $6 \mathrm{~mm}$ in diameter mycelia disks of the test pathogenic fungi in the centre of Petri dishes and then the plates were incubated at $28^{\circ} \mathrm{C}$. Each groups for one concentration was set with three replicates. The diameter of the hyphal colony on each PDA (potato dextrose agar) plate was measured when the hyphae in the control plate reached the edges of plate. The growth inhibition rate was calculated according to the following formula: IR $(\%)=[(\mathrm{GC}-\mathrm{GT}) / \mathrm{GC}]$ $\times 100$, where IR is the inhibition rate and GC and GT represent mycelia growth in the control and treatment, respectively ${ }^{8}$.

The Petri plates of above control group were added distilled water with $0.05 \%$ Tween-80 (v/v) and the spores were collected. $50 \mu \mathrm{L}$ of spore suspension for the test plant pathogenic fungi were added to $5 \mathrm{~mL}$ potato dextrose with the major compound which final concentration was 0.125 , $0.25,0.5,1.0,2.0$ and $4.0 \mathrm{mg} \mathrm{mL}^{-1}$, respectively ${ }^{6}$. The medium without the compound was used as the growth control and all these treatments were incubated in a reciprocating shaker under $28^{\circ} \mathrm{C}, 110 \mathrm{r} / \mathrm{min}$ for $36-48 \mathrm{~h}$. The minimal inhibition concentration (MIC) was defined as the lowest concentration of the antifungal agents that prevented visible growth with respect to the growth control ${ }^{17}$.

Stability of the major compound of F3: Stability of the major compound was tested as described previously by Zhang et al. ${ }^{18}$. Briefly, after the samples were, respectively exposed at $30,40,50,60,70,80,90$ and $100^{\circ} \mathrm{C}$ for $0.5 \mathrm{~h}$, the antifungal activities of the major compound against $F$. graminearum were assayed after the solutions had cooled to room temperature. In order to test the stability of the major compound under acid and alkaline conditions, samples were adjusted to $\mathrm{pH} \mathrm{2,} \mathrm{4,} \mathrm{6,}$ 8,10 using $0.5 \mathrm{M} \mathrm{HCl}$ or $\mathrm{NaOH}$ and then the antifungal activities was determined. The antifungal activities were tested by using growth rate method.

SEM analysis of hyphal ultrastructure: Scanning electron microscope (SEM) was performed to observe the effect of the major compound on mycelia ultrastructure ${ }^{19}$. Mycelium sections of $F$. graminearum, experimental group treated with the major compound solution (final concentration at $0.5 \mathrm{M}$ ) while negative control group only with PBS in same volume, were fixed for $2 \mathrm{~h}$ at $4{ }^{\circ} \mathrm{C}$ by using $2.5 \%$ (v/v) glutaraldehyde in $0.1 \mathrm{M}$ sodium cacodylate buffer after $2 \mathrm{~h}$ of their incubation. After the fixation, the samples were washed three times with $0.1 \mathrm{M}$ cacodylate buffer ( $\mathrm{pH} 7.4$ ), then treated with $1 \%(\mathrm{w} / \mathrm{v})$ osmium tetroxide, washed with $5 \%(\mathrm{w} / \mathrm{v})$ sucrose in cacodylate buffer and subsequently dehydrated in a graded ethanol series (from 30-100\%,). The samples were examined on a JEOL JSM-7500F Scanning Electron Microscopy after the works for critical point drying and gold coating were successfully carried out.

TEM analysis of hyphal ultrastructure: Hyphae sections of $F$. graminearum were firstly incubated for $2 \mathrm{~h}$ in the solution which contained 0.5 M major compound (experimental sample) or in PBS at the same volume (used as the negative control sample), these samples were then fixed for $8 \mathrm{~h}$ at $4{ }^{\circ} \mathrm{C}$, by using $2.5 \%$ (v/v) glutaraldehyde which dissolved in $0.1 \mathrm{M}$ sodium cacodylate buffer. After the fixation finished, they were treated with $1 \%$ phosphotungstic acid. When the cells of samples were gradually desiccated, they were embedded with EPON-812 and their ultrathin sections were prepared according to the standard procedure. The hyphal ultrastructures for experimental and control sample were observed under a FEI Tecnai $\mathrm{G}^{2}$ F20 transmission electronmicroscope after double staining with uranium and plumbum were carried out ${ }^{20}$.

\section{RESULTS AND DISCUSSION}

Based on UV light $(254 \mathrm{~nm})$ analyses, five fractions (F1-F5) were separated from hexane extract of $C$. zedoaria by column chromatography method and their yields were 10.2, $25.3,34.5,8.3$ and $19.0 \%$, respectively. With a mobile phase of hexane-ethyl acetate-acetic acid ( $\mathrm{v} / \mathrm{v}=16: 1: 0.7)$, the $\mathrm{R}_{\mathrm{f}}$ value 
of these five fractions on a silica gel plate were $0.03,0.19$, $0.32,0.58,0.73$, respectively. All of these five fractions could inhibit the mycelium growth of $P$. wasabiae, however, variation in antifungal activity of different fractions was evident and F3 was the most active.

Applying the preparative HPLC (pre-HPLC) system, the compound corresponding to the major peak with retention time 8.8 min was collected and its HRMS (ESI) Mass spectra, FTIR spectra and NMR spectra were analysed while the compound was identified as curcumenol (CAS No. 19431-84-6) (Fig. 1) by compared their spectral data with those reported in the literature ${ }^{21}$.

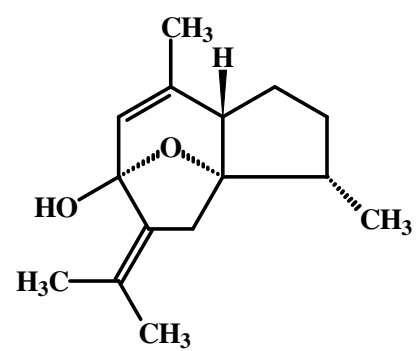

Fig. 1. Structure of curcumenol

The antagonistic activity of curcumenol against the plant pathogenic fungi, including $P$. wasabiae, $F$. graminearum, $P$. brassicae and $M$. grisea, were significant when the concentration is more than $1.0 \mathrm{mg} \mathrm{mL}^{-1}$ (Fig. 2). It showed dosedependent inhibitory activity when the dose increased. The MIC of curcumenol against $F$. graminearum, $P$. wasabiae, $P$. brassicae and $M$. grisea were $0.5,1.0,1.0$ and $1.0 \mathrm{mg} \mathrm{mL}^{-1}$, respectively.
A

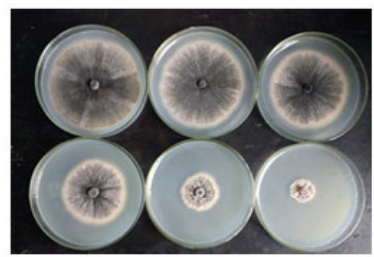

C

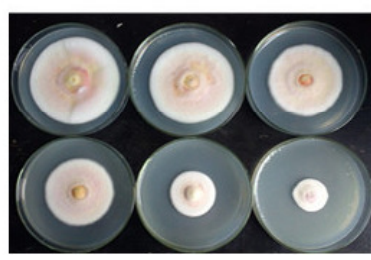

B
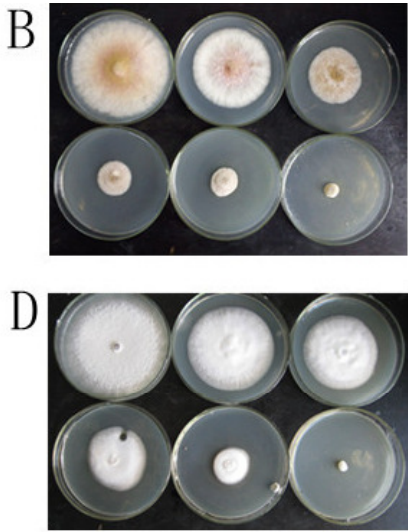

Fig. 2. Effect of curcumenol on different fungal phytopathogens at 125 , 250, 500,1000 and $2000 \mu \mathrm{g} \mathrm{mL} \mathrm{m}^{-1}$; (A) F. graminearum, (B) $P$. wasabiae, (C) P. brassicae

After exposure at $30-70{ }^{\circ} \mathrm{C}$ for $0.5 \mathrm{~h}$, the antifungal activity of curcumenol against $F$. graminearum were not evidently affected. However, it was reduced only $10 \%$ when disposed at $90-100{ }^{\circ} \mathrm{C}$ for $0.5 \mathrm{~h}$. Moreover, curcumenol showed stable antifungal activity because of its activity being not significantly changed when the compound under $\mathrm{pH}$ values from 2 to 10 . All these results suggested that the antifungal activity of curcumenol was stable under a broad temperature and $\mathrm{pH}$ ranges.

The hyphae morphology of $F$. graminearum was also observed under SEM (Fig. 3) in order to compare the mycelium structures of experiment group which treated with the curcumenol solution or without the compound. In the control group (treated without curcumenol), the hyphae hmorphology is intact and their cells were smooth while the experimental group (treated with $0.5 \mathrm{M}$ curcumenol for $2 \mathrm{~h}$ ) showed that the surfaces of mycelium were all developed wrinkled and distorted.
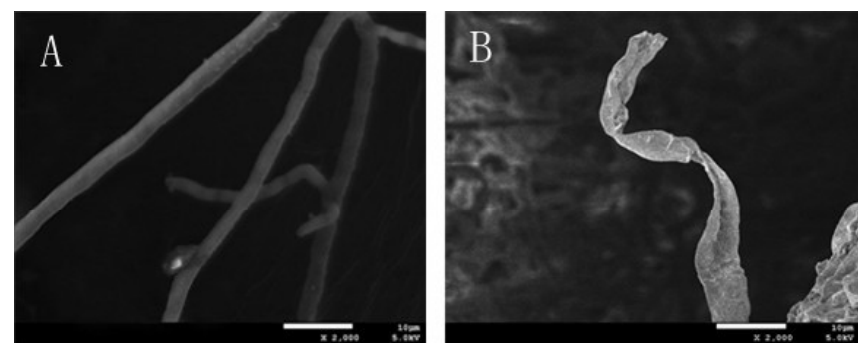

Fig. 3. Scanning electron microscopy (SEM) of F. graminearum; (A) control, (B) cells treated with curcumenol

Results of the TEM photographs (Fig. 4) showed that the cell wall and membrane of $F$. graminearum in which treated with no curcumenol were all intact and clearly seen in their ultrastructure, but that of the curcumenol-treated group, especially the cell-wall exhibited degrading or disappeared, the organelles reducing and disintegrating. There were apparent stripes in intracellular structure of the curcumenol treated group while in the control group cell no similar stripe shape structure was found.
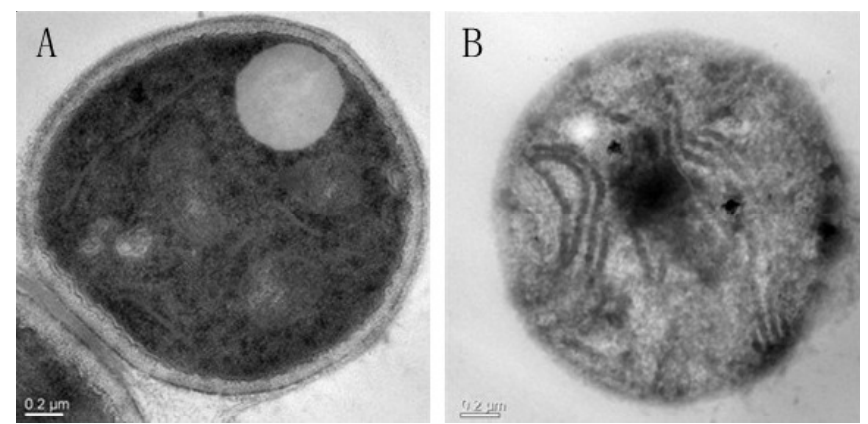

Fig. 4. Transmission electron microscopy (TEM) of F. graminearum; (A) control, (B) cells treated with curcumenol

\section{Conclusion}

In this paper, we purified the compound from C. zedoaria, applying bio-active-guided silica gel column chromatography and high performance liquid chromatography and firstly proved that it had effective antifungal activity against plant pathogenic fungi, such as $P$. wasabiae, F. graminearum, $P$. brassicae and $M$. grisea.

\section{ACKNOWLEDGEMENTS}

The authors are thankful to the financial support provided by Sichuan University under the project of Chengdu Scientific research plan (10GGYB082NC-023, 11DXYB152JH-027) 
and Sichuan province scientific support program (2012ZZ0001). Thanks are also due to Analytical \& Testing Centre of Sichuan University, P.R.China, for the analyses of FTIR, ${ }^{1} \mathrm{H}$ and ${ }^{13} \mathrm{C}$ NMR for samples.

\section{REFERENCES}

1. N. Ameziane, H. Boubaker, H. Boudyach, F. Msanda, A. Jilal and B.A. Ait, J. Agron. Sustain. Dev., 27, 273 (2007).

2. A.G.S. Cuthbertson and A.K. Murchie, J. Crop. Prot., 22, 1125 (2003).

3. V.N. Nguyen, Y.J. Kim, K.T. Oh, W.J. Jung and R.D. Park, J. Biocontrol. Sci. Technol., 17, 1047 (2007).

4. M.M. Gamboa-Angulo, J. Cristóbal-Alejo, I.L. Medina-Baizabal, F. Chí-Romero, R. Méndez-González, P. Simá-Polanco and F. May-Pat, J. Microbiol. Biotechnol., 24, 1955 (2008).

5. O. Berrin, A. Mustafa, O. Ilkay and K. Taner, J. Microbiol. Res., 160, 159 (2005)

6. X.N. Bai, W. Liang, J. Cheng, L.Q. Ma, Y.B. Liu, G.L. Shi, Y.N. Wang and J.C. Gu, J. Inf. Technol. Agric. Eng., 134, 693 (2012).

7. J.D. Zhang, Z. Xu, Y.B. Cao, H.S. Chen, L. Yan, M.M. An, P.H. Gao, Y. Wang, X.M. Jia and Y.Y. Jiang, J. Ethnopharmacol., 103, 76 (2006).

8. V.N. Nguyen, D.M.C. Nguyen, D.J. Seo, R.D. Park and W.J. Jung, J. Biocontrol., 54, 697 (2009).

9. J.L. Mau, E.Y.C. Lai, N.P. Wang, C.C. Chen, C.H. Chang and C.C. Chyau, J. Food Chem., 82, 583 (2003)
10. H. Makabe, N. Maru, A. Kuwabara, T. Kamo and M. Hirota, J. Nat. Prod. Res., 20, 680 (2006).

11. K.I. Kim, J.W. Kim, B.S. Hong, D.H. Shin, H.Y. Cho, H.K. Kim and H.C. Yang, J. Mol. Cells, 10, 392 (2000).

12. Y. Bamba, Y.S. Yun, A. Kunugi and H. Inoue, J. Nat. Med.-Tokyo, 65, 583 (2011)

13. E.Y.C. Lai, C.C. Chyau, J.L. Mau, C.C. Chen, Y.J. Lai, C.F. Shih and L.L. Lin, J. Am. J. Chin. Med., 32, 281 (2004).

14. B. Wilson, G. Abraham, V.S. Manju, M. Mathew, B. Vimala, S. Sundaresan and B. Nambisan, J. Ethnopharmacol., 99, 147 (2005).

15. H.F. Li, Y. Wei, Z.F. Long, Y.Y. Huang and J. Cui, J. Sichuan Univ., 48, 191 (2011).

16. H.H. Liu, Y. Wei, J. Cui, Y.Y. Huang, L.L. Wang, S.G. Liu and Z.F. Long, J. Sichuan Univ., 45, 1235 (2008).

17. I. Ali, F.G. Khan, K.A. Suri, B.D. Gupta, N.K. Satti, P. Dutt, F. Afrin, G.N. Qazi and I.A. Khan, Ann. Clin. Microbiol. Antimicrobol., 9, 7 (2010).

18. T. Zhang, Z.Q. Shi, L.B. Hu, L.G. Cheng and F. Wang, World J. Microbiol. Biotechnol., 24, 783 (2008).

19. J. Zhang, X. Wu and S.Q. Zhang, J. Biotechnol. Lett., 30, 2157 (2008).

20. J.D. Zhang, Z. Xu, Y.B. Cao, H.S. Chen, L. Yan, M.M. An, P.H. Gao, Y. Wang, X.M. Jia and Y.Y. Jiang, J. Ethnopharmacol., 103, 76 (2006).

21. H. Hiroshi, S. Yojiro, N. Seiichiro and T. Tsunematsu, J. Chem. Pharm. Bull., 16, 39 (1968) 